\title{
Lysis of Myotubes by Alloreactive Cytotoxic T Cells and Natural Killer Cells Relevance to Myoblast Transplantation
}

Reinhard Hohlfeld* and Andrew G. Engel

Neuromuscular Research Laboratory and Department of Neurology, Mayo Clinic and Mayo Foundation, Rochester, Minnesota 55905; * Department of Neurology and Department of Neuroimmunology, University of Munich and Max-Planck-Institute for Psychiatry, Munich/Martinsried, West Germany

\begin{abstract}
The aim of this study was to investigate the susceptibility of human myotubes to lysis by the two major types of cytotoxic effector cells, CD3+CD8 + cytotoxic $T$ cells $(C T L)$ and CD16+CD56+ natural killer (NK) cells. The myoblasts preparations used as target cells were $>90 \%$ pure as assessed by immunostaining with the Leu19 monoclonal antibody (MAb) that cross-reacts with the neural cell adhesion molecule N-CAM. Allospecific CTL lines were generated from mixed lymphocyte cultures, and freshly isolated allogeneic and autologous peripheral blood cells were used as a source of NK cells. The cytotoxicity was observed under phase optics and by immunoelectron microscopy, and was quantitated with a chromium release assay. Myotubes were efficiently killed by allospecific CTL and by autologous and allogeneic NK cells. The killing by $C T L$ was inhibited with an anti-class I HLA MAb, and the killing by NK cells was inhibited by depleting peripheral blood cells of CD16+ cells with anti-CD16 MAb and complement. The results have important implications for myoblast transplantation, an experimental therapy of muscular dystrophy. (J. Clin. Invest. 1990. 86:370-374.) Key words: muscular dystrophy • allograft rejection • neural cell adhesion molecule (N-CAM) • major histocompatibility complex • cellmediated cytotoxicity
\end{abstract}

\section{Introduction}

Very little is presently known about the immunologic properties of human myoblasts and myotubes. Such knowledge is essential for myoblast transplantation, a potentially promising experimental therapy for hereditary muscle diseases, such as Duchenne muscular dystrophy (1-3). Myoblast transplantation has been used successfully in a murine model of muscular dystrophy: Injection of normal myoblasts into dystrophic muscle of mdx mice led to myoblast fusion and expression of dystrophin by the mosaic muscle fibers $(2,3)$. One of the potential problems of this therapy is allograft rejection (discussed

Address reprint requests to Dr. Engel, Neuromuscular Research Laboratory, Guggenheim Bldg. G 801, Mayo Clinic, Rochester, MN 55905. Received for publication 27 February 1990.

J. Clin. Invest.

(c) The American Society for Clinical Investigation, Inc.

0021-9738/90/07/0370/05 $\$ 2.00$

Volume 86, July 1990, 370-374 in reference 1). We demonstrate here that cultured human myotubes can be efficiently killed by allospecific CD8+ cytotoxic $\mathrm{T}$ cells $(\mathrm{CTL})^{1}$ and by HLA-nonrestricted natural killer (NK) cells. These findings raise concern that also in vivo, transplanted human myoblasts may be attacked by the two different types of cytotoxic effector cells.

\section{Methods}

MAbs. Anti-CD3 (Leu-4), anti-CD8 (Leu-2a), anti-CD16 (Leu-11b), and anti-CD56 (Leu-19) MAbs were purchased from Becton-Dickinson (Mountain View, CA). Anti-class I HLA MAb W6/32 was obtained from Sera-Lab (Hicksville, NY). For indirect immunofluorescence, biotinylated horse anti-mouse IgG and FITC-labeled avidin-D (both from Vector Laboratories, Burlingame, CA) were used as the secondary reagents. For functional studies, MAbs Leu-11b and W6/32 were freed of azide by dialysis against PBS.

Target cells. K562 cells were obtained from the American Type Culture Collection, Rockville, MD. Human myoblasts and myotubes were cultured as described $(4,5)$. Briefly, the myoblasts were grown in gelatin-coated culture dishes in Ham's F-10 medium containing $20 \%$ heat-inactivated FCS, $0.5 \%$ chick embryo extract (all from Gibco Laboratories, Grand Island, NY), $100 \mathrm{U} / \mathrm{ml}$ penicillin (Sigma Chemical Co., St. Louis, MO), $100 \mu \mathrm{g} / \mathrm{ml}$ streptomycin (Sigma), and $2 \mathrm{mM}$ L-glutamine (Gibco Laboratories). The myoblast preparations used in the experiments were $>90 \%$ pure as assessed by immunostaining with MAb anti-Leu-19 that cross-reacts with the neural cell adhesion molecule N-CAM (6). To induce myotube differentiation, myoblasts were plated in a fusion-supporting medium (Dulbecco's modified Eagle medium containing $2 \%$ heat-inactivated horse serum (both from Gibco), $100 \mathrm{U} / \mathrm{ml}$ penicillin, and $100 \mu \mathrm{g} / \mathrm{ml}$ streptomycin). Under these conditions, fusion was usually complete after 4-5 d.

Effector cells. PBMC were isolated on Ficoll-Hypaque and fractionated into nonadherent PBMC by depleting plastic-adherent and nylon wool-adherent cells (7). Nonadherent PBMC were depleted of NK cells by treatment with anti-Leu- $11 \mathrm{~b}$ and $\mathrm{C}$ as suggested by the supplier. The NK cell-depleted preparations contained no residual Leu-11b or Leu-19-reactive cells as assessed by FACS analysis and fluorescence microscopy. Alloreactive $\mathrm{CD} 3+\mathrm{CD} 8+\mathrm{T}$ cell lines were generated in mixed lymphocyte culture with irradiated allogeneic PBMC and propagated by repeated stimulation with PHA and irradiated allogeneic PBMC (8). The alloreactive CTL lines used in the experiments were $>95 \%$ CD3 +CD8+CD16-CD56- as assessed by flow cytometry. Before use in cytotoxicity assays, the CTL were cultured for at least $7 \mathrm{~d}$ in lectin-free medium.

Flow cytometry. Analysis were performed with a FACS IV system (Becton-Dickinson).

1. Abbreviations used in this paper: CTL, cytotoxic T cells; N-CAM, neural cell adhesion molecule; NK, natural killer cells. 
Cytotoxicity assays. Cytotoxicity against K562 target cells was measured in a standard 4-h ${ }^{51} \mathrm{Cr}$-release assay (7). Cytotoxicity against myotubes was measured in an 8-h ${ }^{51} \mathrm{Cr}$-release assay. Briefly, myoblasts were plated into gelatin-coated, flat-bottomed microwells at 5 $\times 10^{3}$ cells per well. The myoblasts fused into myotubes within 6-8 d of culture. Each well was labeled with $6 \mu \mathrm{Ci}(\mathrm{Na})_{2}{ }^{51} \mathrm{Cr} \mathrm{O}_{4}$ for $1 \mathrm{~h}$, rinsed repeatedly, and the chromium-labeled myotubes were then used as targets. The cytotoxicity assays were done in triplicates. Maximal release was induced with $5 \%$ Triton X-100. Background release ranged from 10 to $24 \%$ of the maximal release. The mean percent specific cytotoxicity, $c$, was calculated for each set of triplicates according to

$$
c=100 \times(x-b) /(m-b)
$$

with $x$, mean experimental release; $b$, mean background release; $m$, mean maximal release.

Morphology. Interactions of living cells in culture were observed under phase optics. A grid of perpendicular lines was drawn on the outer surface of individual wells of a 24-well culture plate (Costar, Cambridge, MA) to help in the identification and sequential observation of individual myotubes. For immunoelectron microscopy, myotubes and CTL were co-cultured on Teflon membranes (9) using a modified ABC immunoperoxidase protocol (10). $1 \mathrm{~h}$ after initiation of co-culture, the cultures were placed on ice and treated with anti-CD8 MAb $(10 \mu \mathrm{g} / \mathrm{ml})$ diluted in PBS containing $2 \%$ BSA, $10 \%$ heat-inactivated serum from $A B+$ donors, and $5 \%$ heat-inactivated horse serum (Gibco). After $30 \mathrm{~min}$ of incubation at $4^{\circ} \mathrm{C}$, the cultures were rinsed three times with ice-cold PBS, treated with $7.5 \mu \mathrm{g} / \mathrm{ml}$ biotinylated horse anti-mouse IgG (Vector Laboratories) for $30 \mathrm{~min}$ at $4^{\circ} \mathrm{C}$, rinsed three times with PBS, fixed with $1 \%$ glutaraldehyde buffered with 0.1 M Tris buffer (pH, 7.6) containing $25 \mathrm{mM}$ hydroxylamine. Next, the cultures were treated with the avidin-biotin peroxidase complex (Vector) for $30 \mathrm{~min}$ at $25^{\circ} \mathrm{C}$, rinsed with PBS for $20 \mathrm{~min}$, treated with $0.01 \%$ hydrogen peroxide, $0.05 \%$ diaminobenzidine in $0.1 \mathrm{M}$ Tris buffer $(\mathrm{pH}$, 7.6) for $15 \mathrm{~min}$ at $25^{\circ} \mathrm{C}$, rinsed with $0.1 \mathrm{M}$ cacodylate buffer $(\mathrm{pH}, 7.5)$ for $30 \mathrm{~min}$, postfixed in $2 \%$ osmium tetroxide for $20 \mathrm{~min}$, reduced with $0.5 \%$ potassium ferrocyanide in $0.1 \mathrm{M}$ cacodylate $(\mathrm{pH}, 7.4)$ for $10 \mathrm{~min}$, rinsed briefly in distilled water, equilibrated with $10 \%$ ethanol for 5 min, stained with $1 \%$ uranyl acetate in $10 \%$ ethanol for $5 \mathrm{~min}$, and then dehydrated with ethanol and infiltrated with Spurr. The Teflon membranes were cut into small $\left(4-5 \mathrm{~mm}^{2}\right)$ pieces that were individually embedded in Spurr. Thick resin sections $(1.5 \mu \mathrm{m})$ were observed in phase optics. Thin sections were cut with diamond knives, stained with lead citrate, and examined in a Philips 400 electron microscope

HLA typing. HLA-ABC typing was carried out on the CTL lines and PBMC using a standard NIH microcytotoxicity assay (Mayo Clinic Blood Bank).

\section{Results}

Cultured myotubes were killed by alloreactive CD3+CD8+ CD16-CD56- T cells (Fig. 1). Lysis involved the recognition of allogeneic class I HLA molecules because it was completely inhibited with anti-class I HLA MAb W6/32 (Fig. 1), and because autologous myotubes were not lysed $(<1 \%$ specific ${ }^{51} \mathrm{Cr}$-release at an E/T ratio of 20:1). Furthermore, alloreactive $T$ cells had no detectable NK activity against K-562 target cells $\left(<1 \%\right.$ specific ${ }^{51} \mathrm{Cr}$-release at an $\mathrm{E} / \mathrm{T}$ ratio of $\left.20: 1\right)$.

We followed the stages of myotube lysis by sequential observation of individual myotubes under phase optics (Fig. 2) The first stage was adhesion of $\mathrm{T}$ cells to myotubes (this became apparent within 30 min after adding the $T$ cells). The second stage was incipient destruction and partial retraction of myotubes $(1 \mathrm{~h})$. The third stage was complete destruction and detachment $(2 \mathrm{~h})$. Immunoelectron microscopic analysis showed that the CD8+ CTL extended CD8+ spikelike projec-

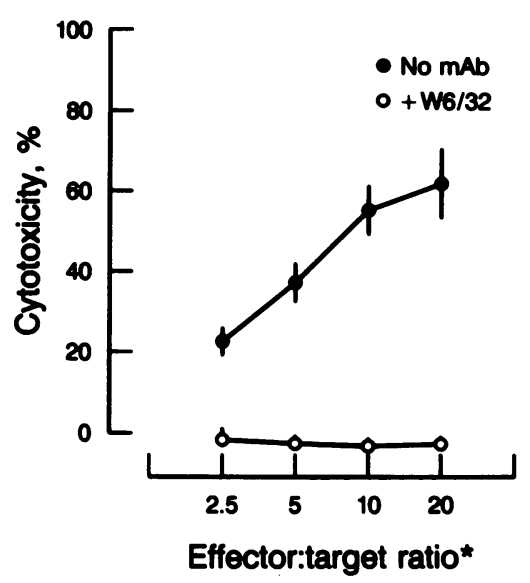

Figure 1. Myocytotoxicity of alloreactive cytotoxic $T$ cells in the presence (open symbols) or absence (solid symbols) of anti-class I HLA MAb W6/32 (50 $\mu \mathrm{g} / \mathrm{ml})$. Cytotoxicity values represent mean \pm SD specific ${ }^{51} \mathrm{Cr}$ release. The HLA class I type of the CTL was A1, 32/B8, 51/Cw7, -, and the HLA class I type of the myotubes was $\mathrm{A} 2,31 / \mathrm{B} 27$, w60/ Cw1, w3.

tions into the target myotubes without obvious lysis of segments of the myotube surface membrane (Fig. 3).

Not only were myotubes susceptible to lysis by alloreactive CTL, but also by freshly isolated allogeneic and autologous PBMC. The lysis of myotubes by PBMC was similar to the lysis of the NK-sensitive target K562 (Fig. 4). The lysis was not due to cytotoxic macrophages because nylon wool-nonadherent, macrophage-depleted cells retained the cytotoxic potential (Fig. 5). In contrast, the cytotoxicity was mediated by CD16+ natural killer cells because it was abrogated by depletion of CD16+ cells with anti-Leu-11b and C (Fig. 5). Expectedly, the lysis of myotubes by PBMC was not inhibited in the presence of anti-HLA class I MAb W6/32 (not shown).

Figs. 4 and 5 show the lysis of myotubes by PBMC from unrelated (allogeneic) donors. We also had the opportunity to test the killing of myotubes by freshly prepared autologous PBMC. At the E/T ratios of 80:1, 40:1, and 20:1, the corresponding cytotoxicity values were $75.8 \pm 3.4,64.8 \pm 6.5$, and $54.4 \pm 1.9$.

\section{Discussion}

In this study we investigated the susceptibility of human myoblasts and myotubes to killing by the two major types of cytotoxic effector cells, CTL and NK cells. Classically, CTL recognizing HLA class I-positive target cells coexpress the CD3 and CD8 differentiation antigens (11). In contrast, the majority of NK cells express an Fc receptor for IgG (CD16) and the CD56 molecule, but are negative for CD3 and CD8 (12). Whereas the killing by CTL is either HLA-restricted or HLA-specific and requires the sensitization of the effector cells against antigen(s) of the target cells, the killing by NK cells is HLA- and antigennondependent and occurs spontaneously, i.e., without prior sensitization (13). Our results demonstrate that human myoblasts and myotubes are susceptible to both CD3+CD8+ allospecific CTL and CD16+CD56+ NK cells. These findings have major implications for myoblast transplantation, an experimental therapy of muscular dystrophy (1-3). In a broader perspective, the findings raise interesting questions regarding the normal regulation of $\mathrm{NK}$ cells in vivo.

Myotubes constitutively express HLA-class I $(5,14)$. This is a necessary but not sufficient precondition for allospecific recognition by CD8 + CTL. For example, mouse trophoblasts (15) and human neuroblastoma cells (16) are resistant to alloreactive lysis even when induced to express high levels of 

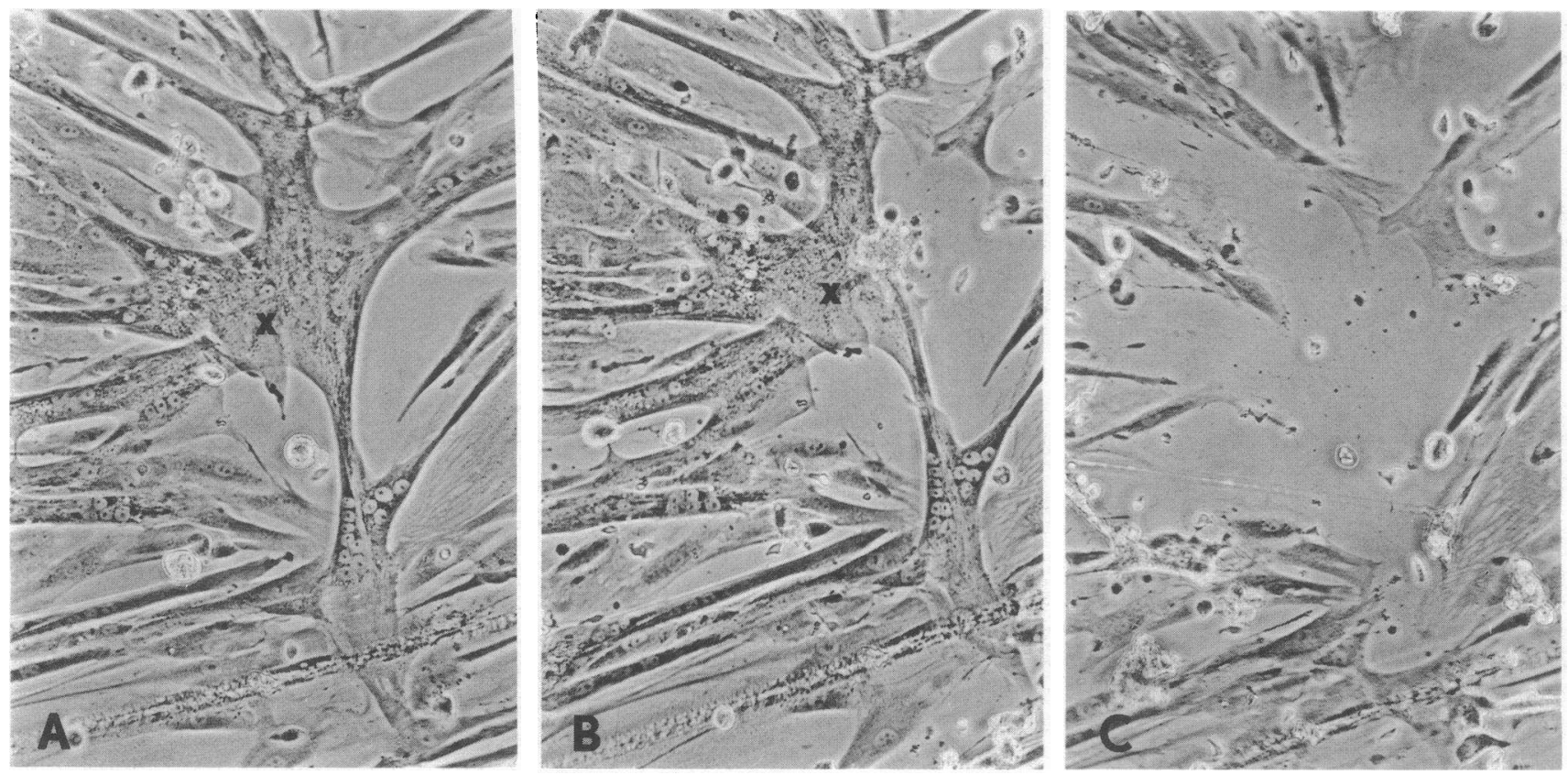

Figure 2. Stages of myotube lysis. Alloreactive cytotoxic $\mathrm{T}$ cells were added at zero time $(A)$. The E/T ratio (based on the number of nuclei) was $\sim$ 1:1. Sequential observation of myotube $(X)$ under phase optics shows its incipient destruction after $1 \mathrm{~h}(B)$, and its complete lysis after $2 \mathrm{~h}$ (C) $(\times 130)$

class I HLA. This may be due to intrinsic resistance to the lytic process (15), or to insufficient expression of accessory molecules (16). Our results indicate that human myotubes express appropriate levels of accessory molecules.
It has been postulated that class I HLA expression is inversely correlated with the susceptibility to NK cell-mediated lysis (17). Several exceptions to this rule have been noted (18, 19). Our observation that class I HLA-positive myotubes are
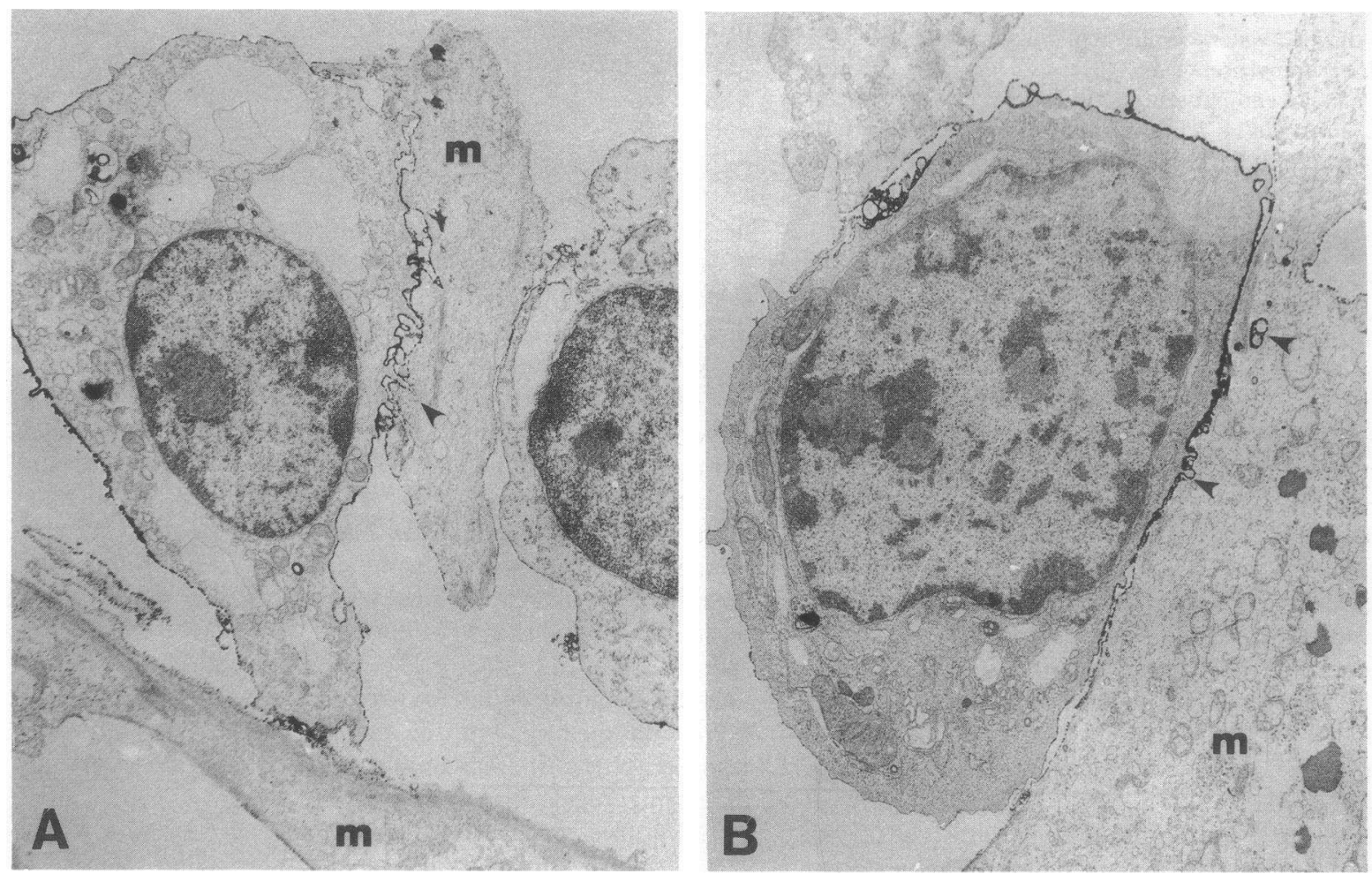

Figure 3. Immunoelectron microscopic visualization of the membrane interaction between a cytotoxic $\mathrm{T}$ cell and a myotube. A CD8+ T cell is attached to a myotube $(m)$ and extends multiple CD8+ spikelike projections into it (arrowheads). The myotube membrane facing the CD8+ cell is stained by diffusion artifact. $(\times 5,000)$ 


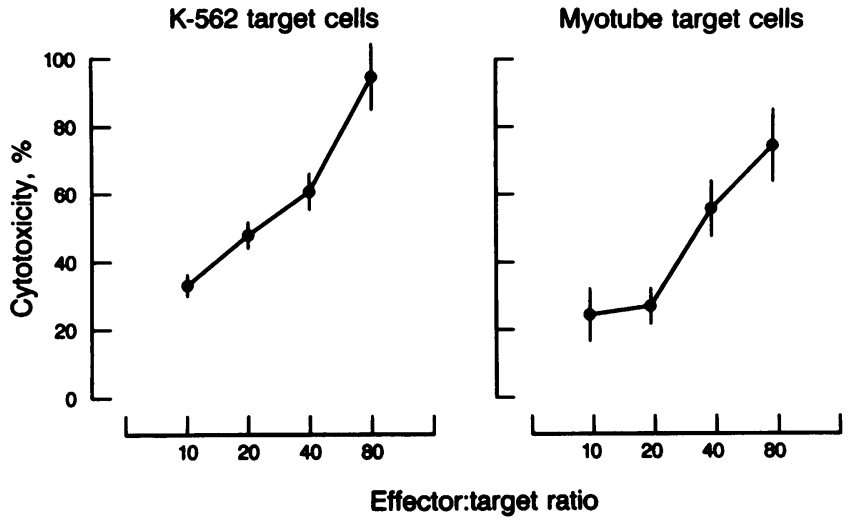

Figure 4. Comparison of the susceptibility to natural killing of K-562 cells $(l e f t)$ and myotubes $(r i g h t)$. Effector cells were freshly isolated PBMC containing 20\% CD16+ cells.

highly susceptible to both types of cytotoxic effector cells represents a further exception. In this regard, it is important that human myotubes react with the anti-Leu-19 MAb (20). This $\mathrm{MAb}$ reacts with CD56, an isoform of the neural cell adhesion molecule N-CAM (6). N-CAM is a homotypic adhesion molecule of the Ig superfamily (21). CD56 is also expressed on NK cells $(12,13)$. There is evidence that CD56 is functionally involved in NK cell-mediated cytotoxicity against targets bearing N-CAM (22). As yet, we have no conclusive evidence that the killing of myotubes by NK cells can be inhibited with anti-CD56 MAb, but this question will be subject to further investigation.

It is not known whether NK cells can attack normal myotubes in vivo. If regenerating myotubes in vivo were as susceptible to NK cell-mediated lysis as are cultured myotubes, normal muscle regeneration could not occur because the myotubes would be killed by autologous NK cells. One possibility is that myotubes are intrinsically more resistant to autologous NK cells in vivo. Regenerating muscle fibers are known to express high levels of $\mathrm{N}-\mathrm{CAM} / \mathrm{CD} 56$ in vivo (20), so that $\mathrm{N}-\mathrm{CAM}$ expression on myotubes is probably not a regulating factor. However, there may be other mechanisms that prevent the lysis of myoblasts and myotubes in vivo. The postulated regulatory factors (e.g., cytokines) may act on the level of effector cells, target cells, or both (reviewed in reference 13).

Our observations have obvious clinical implications for myoblast transplantation, an experimental strategy for the treatment of muscular dystrophy and other hereditary muscle diseases (1-3). Injection of myoblasts from normal mouse or human muscle into growing or regenerating muscle of $\mathrm{mdx}$ mice, which represent a murine genetic homologue of human Duchenne muscular dystrophy $(23,24)$, led to substantial expression and correct localization of dystrophin in the injected muscle (1-3). The transplanted normal myoblasts fused with host muscle fibers giving rise to "mosaic fibers" in which the normal dystrophin gene of the transplanted myonuclei was expressed in muscle fiber segments. Thus, the mosaic fibers were partially rescued from their biochemical defect and escaped fiber necrosis.

Obviously, numerous problems must be dealt with before this promising experimental therapy can be applied in human disease (discussed in reference 1). One of these problems is the possibility of allograft rejection. In one study, rejection was circumvented by using immunocompromised or tolerant host strains (2). However, in another study (3), xenogenic (human) myoblasts fused successfully to muscle fibers of immunocompetent $\mathrm{mdx}$ mice and were apparently not rejected during an observation period of at least $60 \mathrm{~d}$. One possible explanation is that myoblasts, which constitutively express MHC class I antigens (5), lose MHC antigen expression after fusion to mature muscle fibers, which constitutively lack MHC antigens (14). However, even if transplanted myonuclei can transiently evade detection by the host's immune system by losing MHC

\section{Myotube target cells}

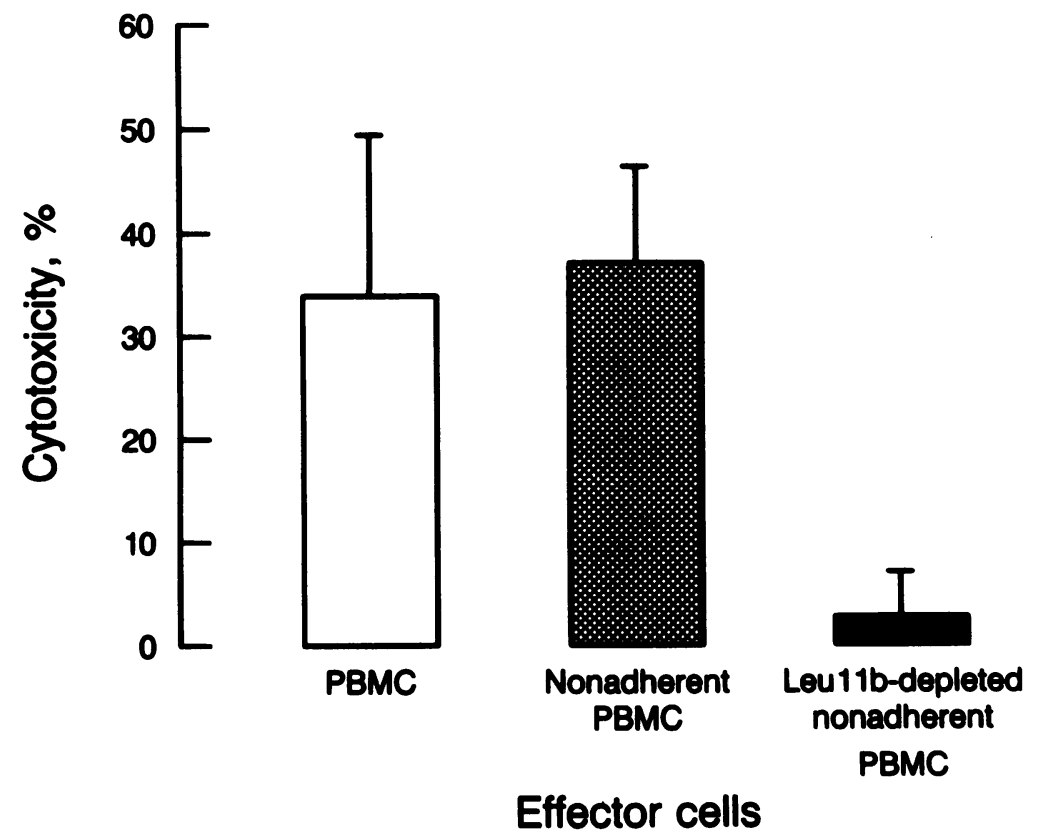

Figure 5. Comparison of the myocytotoxicity of freshly isolated PBMC (left column), plastic- and nylon woolnonadherent PBMC (middle), and Leu-11b-depleted nonadherent PBMC (right). The E/T ratios were 100:1. 
antigen expression, MHC expression could still reappear during fiber regeneration after metabolic, mechanical, or other fiber injury. Our results imply that regenerating muscle fibers, which are the in vivo correlate of cultured myotubes, are likely to be susceptible to lysis by allospecific CTL. Of even more concern is our finding that human myotubes also can be killed by NK cells. Thus, injected myoblasts could be attacked by NK cells in a human host before they could fuse with host myotubes. The conditions under which injected myoblasts can become resistant to NK-cell-mediated killing are presently unknown. The experimental system described here may help in designing strategies relevant to the prevention of myoblast rejection.

\section{Acknowledgments}

We thank Dr. J. A. Katzmann and Ms. T. Kimlinger for help with the flow cytometry, and Dr. H. Wekerle for helpful comments.

This work was supported by NIH grant NS-6277 and a Research Center grant from the Muscle Dystrophy Association. R. H. was the recipient of a Heisenberg grant from the Deutsche Forschungsgemeinschaft.

\section{References}

1. Mandel, J. L. 1989. Dystrophin: The gene and its product. $\mathrm{Na}$ ture (Lond.). 339:584-586.

2. Partridge, T. A., J. E. Morgan, G. R. Coulton, E. P. Hoffman, and L. M. Kunkel. 1989. Conversion of $m d x$ myofibers from dystrophin-negative to positive by injection of normal myoblasts. Nature (Lond.). 337:176-179.

3. Karpati, G., Y. Pouliot, E. Zubrzycka-Gaarn, S. Carpenter, P. N. Ray, R. G. Worton, and P. Holland. 1989. Dystrophin is expressed in mdx skeletal muscle fibers after normal myoblast implantation. Am J. Pathol. 135:27-32.

4. Blau, H. M., and C. Webster. 1981. Isolation and characterization of human muscle cells. Proc. Natl. Acad. Sci. USA. 78:5623-5627.

5. Hohlfeld, R., and A. G. Engel. 1990. Induction of HLA-DR expression on human myoblasts with interferon-gamma. Am. J. Pathol. 136:503-508.

6. Lanier, L. L., R. Testi, J. Binal, and J. H. Phillips. 1989. Identity of Leu-19 (CD56) leukocyte differentiation antigen and neural cell adhesion molecule. J. Exp. Med. 169:2233-2238.

7. Mishell, B. B., and S. M. Shiigi, editors. 1980. Selected Methods in Cellular Immunology. W. H. Freeman Co., San Francisco.

8. Moretta, A., G. Pantaleo, L. Moretta, J.-C. Cerottini, and M. C. Mingari. 1983. Direct demonstration of the clonogenic potential of every human peripheral blood T cell. Clonal analysis of HLA-DR expression and cytoloytic activity. J. Exp. Med. 157:743-754.
9. Gabridge, M. G., and E. P. Dougherty. 1983. Improved method for transmission electron microscopy of ciliated cell monolayers maintained on gas-permeable membranes. J. Microsc. 132:165-172.

10. Arahata, K., and A. G. Engel. 1986. Monoclonal antibody analysis of mononuclear cells in myopathies. III: Immunoelectron microscopy aspects of cell-mediated muscle fiber injury. Ann. Neurol. 19:112-125.

11. Royer, H. D., and E. L. Reinherz. 1987. T-lymphocytes: ontogeny, function, and relevance to clinical disorders. N. Engl. J. Med. 317:1136-1142.

12. Lanier, L. L., A. M. Le, C. I. Civin, M. R. Loken, and J. H. Phillips. 1986. The relationship on CD16 (Leu-11) and Leu-19 (NKH-1) antigen expression of human peripheral blood NK cells and cytotoxic T lymphocytes. J. Immunol. 136:4480-4486.

13. Trinchieri, G. 1989. Biology of natural killer cells. Adv. Immunol. 47:187-376.

14. Karpati, G., Y. Pouliot, and S. Carpenter. 1988. Expression of immunoreactive major histocompatibility complex products in human skeletal muscles. Ann. Neurol. 23:64-72.

15. Zuckerman, F. A., and J. R. Head. 1987. Murine trophoblasts resist cell-mediated lysis. I: Resistance to allospecific cytotoxic T lymphocytes. J. Immunol. 139:2856-2864.

16. Main, E. K., D. S. Monos, and L. A. Lampson. 1988. IFNtreated neuroblastoma cell lines remain resistant to $\mathrm{T}$ cell-mediated allo-killing, and susceptible to non-MHC-restricted cytotoxicity. $J$. Immunol. 141:2943-2950.

17. Karre, K., H. G. Ljunggren, G. Piontek, and R. Kiessling. 1986. Selective rejection of H-2-deficient lymphoma variants suggests alternative immune defence strategy. Nature (Lond.). 319:675-678.

18. Chervenak, R., and R. M. Walcott. 1988. Target cell expression of MHC antigens is not (always) a turn-off signal to natural killer cells. J. Immunol. 140:3712-3716.

19. Leiden, J. M., B. A. Karpinski, L. Gottschalk, and J. Kornbluth. 1989. Susceptibility to natural killer cell-mediated cytolysis is independent of the level of target cell class I HLA expression. J. Immunol. 142:2140-2147.

20. Schubert, W., K. Zimmermann, M. Cramer, and A. Starzinski-Powtiz. 1989. Lymphocyte antigen Leu-19 as a molecular marker of regeneration in skeletal muscle. Proc. Natl. Acad. Sci. USA. 86:307-311.

21. Edelman, G. M. 1987. CAMs and Igs: Cell adhesion and the evolutionary origins of immunity. Immunol. Rev. 100:11-45.

22. Nitta, T., H. Yagita, K. Sato, and K. Okumura. 1989. Involvement of CD56 (NKH-17/Leu-19 antigen) as an adhesion molecule in natural killer-target cell interaction. J. Exp. Med. 170:1757-1761.

23. Hoffman, E. P., R. H. Brown, and L. M. Kunkel. 1988. Dystrophin: The protein product of the Duchenne muscular dystrophy locus. Cell. 51:919-928.

24. Gutmann, D. H., and K. H. Fischbeck. 1989. Molecular biology of Duchenne and Becker's muscular dystrophy: clinical applications. Ann. Neurol. 26:189-194. 